\title{
A Monitor Function for Spectral Moving Mesh Methods Applied to Nonlinear Acoustics
}

\author{
Elliott S. Wise ${ }^{\text {a) }}$, Ben T. Cox and Bradley E. Treeby \\ Department of Medical Physics and Biomedical Engineering, University College London, United Kingdom \\ ${ }^{a)}$ Corresponding author: elliott.wise.14@ucl.ac.uk
}

\begin{abstract}
Spectral methods have made linear acoustics simulations highly computationally efficient, but they currently lose their efficiency when modelling nonlinear waves with regular grids. Moving mesh methods can address this by distributing mesh nodes to minimise the number of nodes needed to represent a waveform. In this paper, a monitor function is presented which is designed specifically for spectral moving mesh methods. In comparison with past monitor functions, this new monitor function significantly improves the convergence rate of the spectral moving mesh method when applied to Burgers' equation.
\end{abstract}

\section{INTRODUCTION}

The full-wave simulation of nonlinear wave propagation is a computationally challenging task. This is due to the formation of high-frequency harmonics at shock fronts which require resolution by dense computational grids [1]. Spectral methods have proved to be effective in minimising the size of computational grids when compared with finite difference or finite element methods, but until now simulations have been limited to fixed or equispaced meshes. Moving mesh methods adaptively adjust the location of mesh nodes to decrease the error between the approximate solution given by a numerical method and the true solution to a model's governing equations. A monitor function is used to control the density of nodes, and their trajectories are controlled using a moving mesh partial differential equation (MMPDE). Figure 1 shows a flowchart illustrating how a moving mesh method can be used in the numerical solution of a partial differential equation (PDE). Moving mesh methods are well established for use in conjunction with finite difference methods, but there has been limited exploration of their use with spectral methods [2, 3]. This paper focusses on the development of a monitor function which is specifically designed to improve the efficiency of spectral methods.
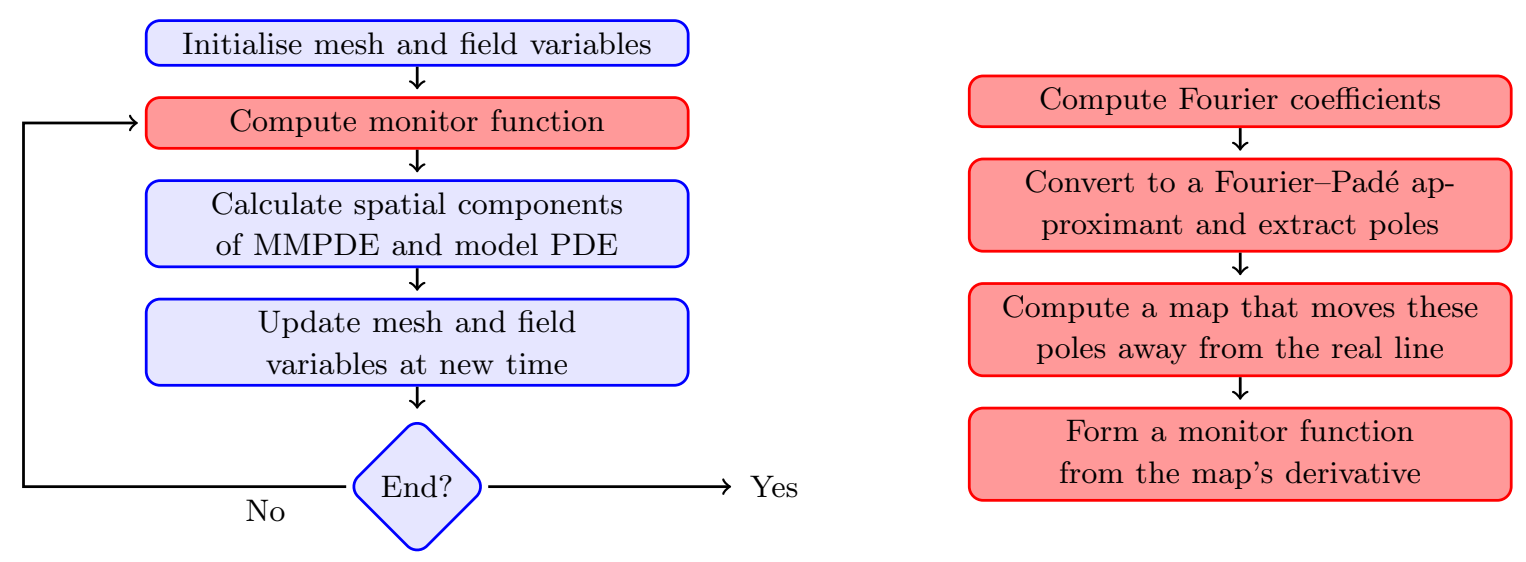

FIGURE 1. Flowcharts illustrating key steps in the algorithms used in this paper. (Left) Structure of a moving mesh method applied to the solution of a PDE. (Right) Steps required to compute a spectral monitor function. 


\section{SPECTRAL CONVERGENCE AND MONITOR FUNCTIONS}

This work uses a numerical method consisting of a rational trigonometric spectral method in space and an implicit Runge-Kutta method in time, though the principles discussed extend easily to other spectral methods. The rational trigonometric spectral method is useful because it is able to perform spectral differentiation on arbitrary meshes. For a set of values $f_{n}$ defined at $N$ arbitrary nodes $x_{n}$, the rational trigonometric interpolant [4] can be written as

$$
r(x)=\frac{\sum_{n=0}^{N-1}(-1)^{n} \operatorname{cst}\left(\frac{x-x_{n}}{2}\right) f_{n}}{\sum_{n=0}^{N-1}(-1)^{n} \operatorname{cst}\left(\frac{x-x_{n}}{2}\right)}, \quad \operatorname{cst} x:= \begin{cases}\csc x, & \text { if } N \text { is odd }, \\ \cot x, & \text { if } N \text { is even } .\end{cases}
$$

For equispaced points $x_{n}$, this interpolant simplifies to a trigonometric polynomial with a characteristically fast convergence rate. If $x_{n}$ are not equispaced then the interpolant is a quotient of two trigonometric polynomials and will not, in general, converge quickly. However, for the special case where $x_{n}$ can be generated by a conformal map $g$ from a set of equispaced points $s_{n}$, the fast convergence property is preserved. More explicitly, for a periodic function $f$, the rational trigonometric interpolant (sampled at $N$ points $x_{n}=g\left(s_{n}\right)$ with $s$ equispaced and $g$ conformal) converges on $f$ at a rate which depends on smoothness. Denoting function composition with $\circ$, the convergence rate is either

Algebraic: $\quad O\left(N^{-p}\right)$ if $f \circ g$ has simple jump discontinuities in the $p$-th derivative, or Geometric: $\quad O\left(e^{-\eta N}\right)$ if $f \circ g$ can be analytically continued into the complex strip $\{z \in \mathbb{C}:|\operatorname{Im} z| \leq \eta\}$.

For the geometric convergence rate, analytic continuation refers to the process of taking a function which is analytic (and hence infinitely differentiable) and defined in a particular domain (such as the real line) and extending its definition to a larger domain in which it remains analytic. The parameter $\eta$ then refers to the widest complex strip in which $f \circ g$ remains analytic. Restricting attention to functions which are analytic on the real line, the task of a spectral moving mesh method should thus be to generate a mesh given by $x=g(s)$ with $s$ equispaced such that the $\eta$ corresponding to $f \circ g$ is greater than that corresponding to $f$ alone.

For the piecewise polynomial interpolants used in finite difference methods, monitor functions are often based on spatial derivatives. Specifically, for a degree $k$ piecewise polynomial interpolant, there exists an error bound related to the modelled quantity's $(k+1)$-th spatial derivative [5, pp. 54-64]. For a mesh with $N$ nodes in each dimension, a spectral polynomial interpolant will be of degree $N$, which suggests that an appropriate monitor function should use the $(N+1)$-th spatial derivative. However, computing these derivatives is numerically difficult, as ill-conditioning results in rounding errors accumulating rapidly as the differentiation order increases [6]. If the spectral method uses a trigonometric polynomial interpolant, it is unclear whether the previously mentioned error bounds even apply. Thus, despite their past use in a spectral context [2,3], derivative-based monitor functions are likely to be non-optimal for spectral moving mesh methods.

Tee et al. $[7,8,9,10]$ devised an approach for finding an optimal mesh more naturally related to spectral methods: they compute a conformal map under which the solution's analytic strip is widened. We extend their method to improve its robustness and general applicability, and implement it within a classical moving mesh framework.

\section{A SPECTRAL MONITOR FUNCTION}

The first step in the method of Tee et al. is to determine where the approximated function $f$ is analytic. This is done by considering where $f$ is not analytic, that is, where the continuation of $f$ has singularities or branch cuts. We use a Fourier-Padé approximant for this, which approximates singularities and branch cuts using singular points known as poles. With a few caveats, the Padé approximant is known to converge on $f$ throughout the complex plane aside from at the singularities of $f$ themselves, where the Padé approximant will have its own poles.

The Fourier-Padé approximant is found in several steps. First, the sampled function $f_{n}$ is evaluated using Eq. (1) at a set of equispaced nodes with a density equal to the maximum density of points for the current mesh. Fourier series coefficients are then computed using the fast Fourier transform. Denoting the Fourier coefficients by $a_{k}$ and $a_{-k}$ and letting $k$ denote wavenumbers, this series can be expressed in a split, exponential form as

$$
f(x)=\sum_{k} a_{k} e^{i k x}+\sum_{k} a_{-k} e^{-i k x}=\sum_{k} a_{k} z_{+}^{k}+\sum_{k} a_{-k} z_{-}^{k} .
$$


Each of these sums can be thought of as a power series about the origin under the coordinate transformations $z_{ \pm}=e^{ \pm i x}$. A Fourier-Padé approximant $f_{F P}$ is then defined as a sum of Padé approximants to each of these power series'

$$
f_{F P}(x)=\frac{p_{1}\left(z_{+}\right)}{q_{1}\left(z_{+}\right)}+\frac{p_{2}\left(z_{-}\right)}{q_{2}\left(z_{-}\right)}, \quad \text { with } \quad \frac{p_{1}\left(z_{+}\right)}{q_{1}\left(z_{+}\right)} \approx \sum_{k} a_{k} z_{+}^{k}, \quad \frac{p_{2}\left(z_{-}\right)}{q_{2}\left(z_{-}\right)} \approx \sum_{k} a_{-k} z_{-}^{k} .
$$

Here, $p$ and $q$ are themselves power series' whose order can be set to specify accuracy in the Padé approximant. The robust Padé approximation algorithm of Gonnet et al. [11] is used for computing $p$ and $q$, since it adaptively adjusts the orders of the approximant to remove spurious poles whose effect is cancelled out by nearby zeroes. The inverse coordinate transformation $x=\mp i \ln z_{ \pm}$is then used to convert the poles of each Padé approximant (zeroes of $q_{1}$ and $q_{2}$ ) into the poles of the Fourier-Padé approximant.

The next step is to find a conformal map $g$ which moves the poles of $f$ away from the computational interval. Following Tee et al., we compute a Schwarz-Christoffel mapping from slits starting at each pole and extending out to $\pm i \infty$ onto the edges of a single strip. This mapping squeezes analyticity in $f$, that would otherwise be lost in a spectral method, into the widest analytic strip of $f \circ g$. Figure 2 gives an illustration of this.

Lastly, the equidistribution condition [5, p. 29] is used to form a monitor function $\rho$ from $g$. This condition states that the integral of $\rho$ should be equal between all adjacent mesh nodes $x_{n}$, with this condition being met by a MMPDE as it converges. The monitor function $\rho=1 / g^{\prime}\left(s_{n}\right)$ can be shown to meet the equidistribution condition when $x_{n}=g\left(s_{n}\right)$ with $s_{n}$ equispaced, that is, when the MMPDE has converged on the desired optimal mesh.

\section{APPLICATION TO BURGERS' EQUATION}

Burgers' equation is a simplification of the full-wave nonlinear Westervelt equation assuming one-way wave propagation. The analytic continuation of the solution to Burgers' equation is multivalued, with the principal branch containing branch points (with corresponding cuts extending outwards to $\pm i \infty$ ) which approach the real line as shocks form [12]. To assess the performance of the spectral monitor function, we solve the initial value problem

$$
u_{t}=0.1 u_{x x}+u u_{x}, \quad u(x, 0)=\sin x, \quad 0 \leq t \leq 1.618839,
$$

for which Cole [13] provides an exact solution. The termination time here was chosen to approximate the time at which the slope at the shockfront is maximised.

Figure 2(a) shows Cole's solution $u$ at the termination time, along with a numerical analytic continuation. A string of poles can be seen at the shock front along the branch cut, indicated by the solid red line. The widest analytic strip of $u$ is the region between the red dashed lines. A conformal map $g$ is then generated from the approximate poles, giving the composed function $u \circ g$ and its continuation shown in Fig. 2(b). The poles of $u \circ g$ now appear on the edges of a single strip, indicated with a solid red line, whose width has increased by a factor of approximately 4.02.

To apply a moving mesh method to this problem, the model is adjusted to account for mesh movement, and a MMPDE is added:

$$
u_{t}=0.1 u_{x x}+u u_{x}+u_{x} x_{t}, \quad x_{t}=\tau^{-1}\left(\rho x_{s}\right)_{s}, \quad \tau=10^{-2} .
$$

Here $\rho$ is the monitor function, computed as previously described, $\tau$ controls the mesh speed, and $s$ is a set of equispaced points. This problem was solved numerically using the spectral moving mesh method with four monitor functions: uniform (no mesh movement), arclength (first spatial derivative), curvature (second spatial derivative) [5, pp. 65-66], and the new spectral monitor function. Figure 2(c) compares the performance of each of these, with the error computed as the infinity-norm between the true and approximated solutions. The derivative-based monitor functions appear to provide limited improvement in performance. The spectral monitor function, however, appears to give geometric convergence at a rate which is significantly faster. Using this monitor function, the exponent in the convergence rate has improved by a factor of approximately 3.2 over the uniform method. This means that for a given accuracy, one might expect to use one third the number of mesh nodes as in a fixed spectral method.

\section{CONCLUSION}

In this paper, a spectral moving mesh method is described based on rational trigonometric interpolants. A spectral monitor function has been designed for the spectral moving mesh methods with this interpolant in mind. When applied to Burgers' equation, the spectral monitor function drastically improves the rate of convergence of the spectral moving mesh method when compared with both a static spectral method and with previously used monitor functions. 

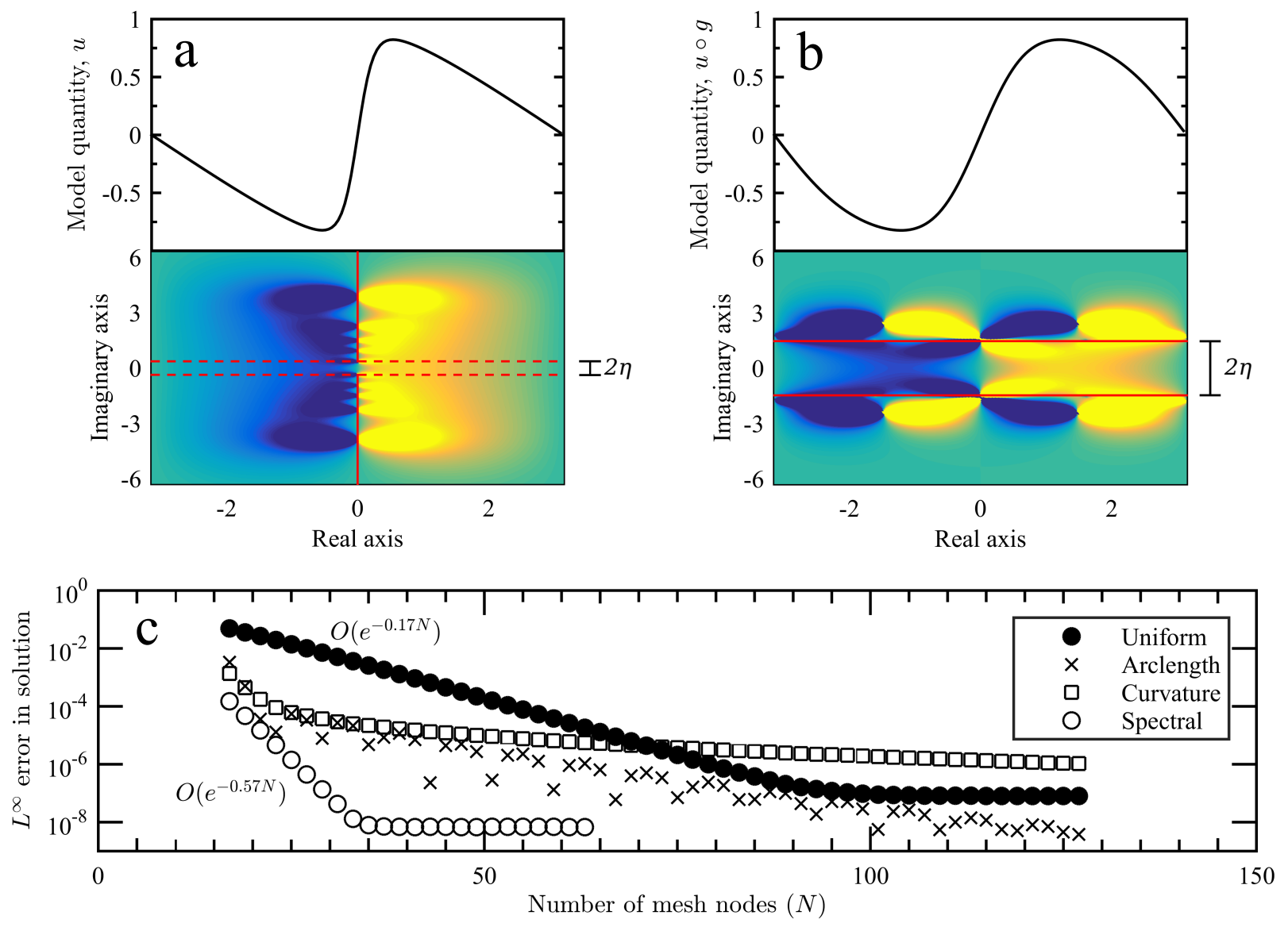

FIGURE 2. (a) Cole's solution $u$ to the initial value problem given by Eq. (4) and an analytic continuation of this solution beyond the real line. (b) Composition of $u$ with a conformal map $g$ generated from the approximate poles of $u$, along with its analytic continuation beyond the real line. (c) Comparison of a number of monitor functions applied to solving Eq. (5), showing that the spectral monitor function leads to much faster convergence than the derivative-based monitor functions.

\section{REFERENCES}

[1] J. Jaros, A. P. Rendell, and B. E. Treeby, Int. J. High Perform. Comput. Appl. (2015).

[2] W. Feng, P. Yu, S. Hu, and Z. Liu, Commun. Comput. Phys. 5, 582-599 (2009).

[3] J. Shen and X. Yang, J. Comput. Phys. 228, 2978-2992 (2009).

[4] R. Baltensperger, Comput. \& Math. with Appl. 43, 737-746 (2002).

[5] W. Huang and R. D. Russell, Adaptive moving mesh methods, Vol. 174 (Springer Science \& Business Media, 2010).

[6] F. Bornemann, Found. Comput. Math. 11, 1-63 (2010).

[7] T. W. Tee and L. N. Trefethen, SIAM J. Sci. Comput. 28, 1798-1811 (2006).

[8] T. W. Tee, "An adaptive rational spectral method for differential equations with rapidly varying solutions," Ph.D. thesis, University of Oxford 2006.

[9] N. Hale and T. W. Tee, SIAM J. Sci. Comput. 31, 3195-3215 (2009).

[10] N. Hale, "On The Use Of Conformal Maps To Speed Up Numerical Computations," Ph.D. thesis, University of Oxford 2009.

[11] P. Gonnet, R. Pachón, and L. N. Trefethen, Electron. Trans. Numer. Anal. 38, 146-167 (2011).

[12] J. A. C. Weideman, SIAM J. Appl. Dyn. Syst. 2, 171-186 (2003).

[13] J. D. Cole, Q. Appl. Math. 9, 225-236 (1951). 